\title{
Quantitative pre-treatment EEG prediction of therapeutic response in schizophrenic and depressive patients
}

Andrey F. Iznak1, Ekaterina V. Iznak1, Elena V. Damyanovich1, and Igor V. Oleichik2

1 Laboratory of Neurophysiology, Mental Health Research Center, Moscow, Russia; e-mail: iznak@inbox.ru

2 Department of Endogenous Mental Disorders and Affective Conditions, Mental Health Research Center, Moscow, Russia

Introduction: Optimization of treatment of endogenous mental disorders by early prediction of therapeutic response is actual due to their heavy social-economic burden and high percent of non-responders.

Objectives: The study aimed to search for some pretreatment EEG parameters informative for individual quantitative prediction of therapeutic response in schizophrenic and depressive patients.

Patients and Methods: 50 female in-patients suffered from attack-like paranoid schizophrenia with affectivedelusional disorders, and 30 female depressive inpatients were included in the study. Resting eye closed EEGs were registered before the beginning of the treatment course, and absolute EEG spectral power values were measured. Quantitative clinical assessments (by PANSS and HDRS rating scales) were obtained after treatment course. Pre-treatment EEG data were matched with post-treatment PANSS and HDRS scores of the same patients using correlation and multiple regression analyses.

Results: Multiple regression equations obtained contained only 3 to 4 EEG spectral power parameters (from initial input of 80 ) and free term. They explained up to $87 \%$ of post-treatment PANSS scores variance (both PANSS-sum, and PANSS-positive, and PANSS-negative subscales, separately) in schizophrenic patients, and up to $75 \%$ of post-treatment HDRS scores variance (of HDRS depression cluster) in depressive patients. Differences between predicted and really obtained posttreatment PANSS scores varied from $3 \%$ to $25 \%$ (mean $10 \%$ ) in schizophrenic patients, and from $10 \%$ to $37 \%$ (mean 24\%) of HDRS scores in depressive patients.
Conclusions: The data obtained may contribute to development of novel methods for quantitative individual prediction of therapeutic response in schizophrenic and depressive patients.

Key words: schizophrenia, depression, pre-treatment EEG, quantitative prediction of therapeutic response

Acknowledgements: The study supported from Russian Basic Research Foundation Grant No.18-01-00029a 